\title{
lon-induced nucleation: A density functional approach
}

\author{
I. Kusaka, Z.-G. Wang, and J. H. Seinfeld \\ Department of Chemical Engineering, California Institute of Technology, Pasadena, California 91125
}

(Received 4 August 1994; accepted 4 October 1994)

\begin{abstract}
Density functional theory is applied to ion-induced nucleation of dipolar molecules. The predicted reversible work shows a sign preference, resulting in a difference in the nucleation rate by a factor of $10-10^{2}$, for realistic values of model parameters. The sign effect is found to decrease systematically as the supersaturation is increased. The asymmetry of a molecule is shown to be directly responsible for the sign preference in ion-induced nucleation. (c) 1995 American Institute of Physics.
\end{abstract}

\section{INTRODUCTION}

The presence of ions has been shown to greatly enhance the rate of nucleation of liquid drops in a supersaturated vapor. $^{1-6}$ The phenomenon of ion-induced nucleation plays an important role in atmospheric condensation, particularly in the ionosphere. While both positive and negative ions increase the nucleation rate, a variety of substances exhibit a dependence of the nucleation rate on the sign of the ion. ${ }^{1-6}$ No theory currently exists that is capable of predicting this sign dependence.

One of the key quantities in estimating a nucleation rate is the reversible work to form a critical nucleus from a metastable state. Unlike the case of homogeneous nucleation, the metastable state relevant here is identified as the one with a vapor-solvated ion. The earliest attempt to calculate the reversible work of ion-induced nucleation is due to Thomson, ${ }^{7}$ based on the theory of capillarity, where a nucleus, either critical or metastable, is represented as a bulk liquid enclosed by an interface of zero thickness with an ion placed at the center. The reversible work is then given in terms of the thermodynamic quantities such as the surface tension, dielectric constants of the bulk phases, etc. The reversible work depends on $q^{2}$, where $q$ is the ion charge, and has no dependence on the sign of $q$.

Physically, the dependence of the ion-induced nucleation rate of a substance on the sign of the ion charge must arise from some asymmetry in the molecular interactions. Such asymmetry should, in principle, manifest itself in a sign dependence of the relevant thermodynamic quantities such as the surface tension. Several attempts have been made to incorporate molecular characteristics within the framework of the capillarity theory, ${ }^{4,8,9}$ where a certain structure in the interfacial region is inevitably assumed. A somewhat different approach was taken by Rusanov and Kuni, ${ }^{10,11}$ in which the surface tension is related to the ion charge and the distance between two dividing surfaces located in the interfacial region. The reversible work predicted by the theory shows extreme sensitivity to this distance, ${ }^{12}$ a slight change of which results in a reversal of the sign preference in the nucleation rate. It has also been noted ${ }^{12}$ that, except for its inability to explain the sign effect, the best predictions of the reversible work, when compared with experimental data, come from the original Thomson's equation.

Clearly, even a qualititative description of ion-induced nucleation requires a theory based on a statistical mechanical treatment, which assumes an intermolecular potential as the fundamental information required to evaluate the relevant thermodynamic potentials. One of such approaches is to directly evaluate the partition function of a nucleus by Monte Carlo simulation. Recently, major progress has been made in this direction for homogeneous nucleation by Reiss and co-workers. ${ }^{13-15}$ An alternative, and computationally less demanding, approach is to use density functional theory. The theory was first applied to homogeneous nucleation in a onecomponent system by Oxtoby and Evans. ${ }^{16}$ In this theory, the grand potential $\Omega$ (equal to $-p V$ for a uniform system) is written as a functional of order parameters such as the average number density of molecules. The critical nucleus is obtained from the stationarity condition of the grand potential with respect to the density profile. Recently, the theory has also been applied to binary homogeneous nucleation. ${ }^{17}$

In this work, we present a density functional theory for ion-induced nucleation of dipolar molecules. Asymmetry is introduced into a molecule by placing a dipole moment at some fixed distance from its center. As a result of the asymmetric nature of the molecules and their interactions with the ion, the reversible work acquires a dependence on the ion charge.

The outline of this paper is as follows. In Sec. II, we first briefly review some of the important results from the general framework of density functional theory, and then develop a density functional for the grand potential in terms of two order parameters, the number density of molecules, and the average dipole moment. Bulk properties are derived from the density functional. Section III describes the solution methods to determine the equilibrium profiles. The reversible work of nucleation is obtained from the equilibrium profiles and reported in Sec. IV. Finally, some concluding remarks are given with a brief discussion in Sec. V.

\section{DENSITY FUNCTIONAL THEORY}

\section{A. The model and some general results}

Let us consider a system of spherical molecules each of which has a dipole of moment $p_{0}$ located at some fixed distance $a$ from its center. For simplicity, the dipole moment is assumed to be pointing in the radial direction with respect to the center of the molecule, as shown in Fig. 1. Suppose that 


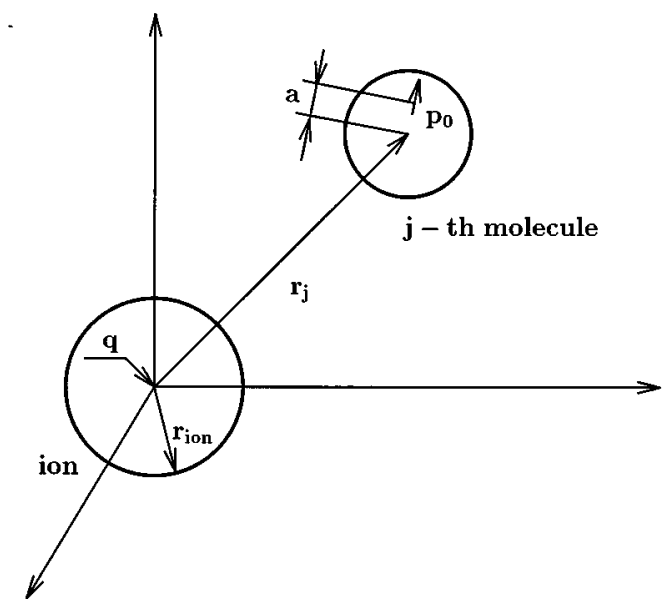

FIG. 1. Model of a dipolar molecule and an ion. The molecule has a dipole moment $p_{0}$ pointing outward with respect to its center at a distance $a$ from the center. An ion is represented as a point charge placed at the center of a hard sphere of radius $r_{\text {ion }}$.

the interaction potential $\phi(1,2)$ between one molecule at $\mathbf{r}_{1}$ with orientation $\hat{R}_{1}$ and the other at $\mathbf{r}_{2}$ with orientation $\hat{R}_{2}$ can be written as

$$
\phi(1,2)=\phi^{d}\left(r_{12}\right)+\phi^{\mathrm{att}}\left(r_{12}\right)+\phi^{p}(1,2),
$$

where $r_{12}=\left|\mathbf{r}_{2}-\mathbf{r}_{1}\right|$. We use the notation $j$ to represent both the translational and orientational coordinates of molecule $j$. $\phi^{d}\left(r_{12}\right)$ is the hard sphere potential given by

$$
\phi^{d}\left(r_{12}\right)=\left\{\begin{array}{cc}
+\infty, & \text { if } r_{12}<d \\
0, & \text { otherwise }
\end{array} .\right.
$$

$\phi^{\text {att }}\left(r_{12}\right)$ is the perturbative attractive potential whose explicit form is chosen to be

$$
\phi^{\mathrm{att}}\left(r_{12}\right)=-\epsilon\left(\frac{d}{r_{12}}\right)^{6},
$$

where $\epsilon$ is a positive constant. $\phi^{p}(1,2)$ is the dipole-dipole interaction between molecules 1 and 2, and is given by

$$
\phi^{p}(1,2)=\frac{p_{0}^{2}}{u_{12}{ }^{3}}\left[\hat{R}_{1} \cdot \hat{R}_{2}-3\left(\hat{R}_{1} \cdot \hat{u}_{12}\right)\left(\hat{R}_{2} \cdot \hat{u}_{12}\right)\right],
$$

where $\mathbf{u}_{j}=\mathbf{r}_{j}+a \hat{R}_{j}, u_{12}=\left|\mathbf{u}_{2}-\mathbf{u}_{1}\right|$, and $\hat{u}_{12}=\left(\mathbf{u}_{2}-\mathbf{u}_{1}\right) / u_{12}$. To avoid complications due to possible overlap of two dipoles, we choose $a$ to be smaller than $d / 2$.

Consider an open system, for which the grand potential $\Omega$ is the proper thermodynamic potential. In density functional theory, $\Omega$ of the system is given as a functional of order parameters such as the position-orientation distribution function $\rho(\mathbf{r}, \hat{R})$ defined by

$$
\rho(\mathbf{r}, \hat{R}) \equiv\left\langle\sum_{j=1}^{N} \delta\left(\mathbf{r}-\mathbf{r}_{j}\right) \delta\left(\hat{R}-\hat{R}_{j}\right)\right\rangle .
$$

Later, we shall use the particle density distribution function

$$
n(\mathbf{r}) \equiv\left\langle\sum_{j=1}^{N} \delta\left(\mathbf{r}-\mathbf{r}_{j}\right)\right\rangle
$$

and the orientation distribution function

$$
m(\mathbf{r}, \hat{R}) \equiv \rho(\mathbf{r}, \hat{R}) / n(\mathbf{r}) .
$$

From the definition Eqs. (5)-(7), it follows that

$$
n(\mathbf{r})=\int d \hat{R} \rho(\mathbf{r}, \hat{R})
$$

and

$$
\int d \hat{R} m(\mathbf{r}, \hat{R})=1 .
$$

In terms of $\rho(\mathbf{r}, \hat{R}), \Omega$ is given exactly by ${ }^{18}$

$$
\begin{aligned}
\Omega[\rho]= & F_{r}[\rho]-\int d 1 \rho(1)[\mu-v(1)] \\
& +\frac{1}{2} \iint d 1 d 2 \rho(1) \rho(2) \int_{0}^{1} d \lambda g(1,2 ; \lambda) \\
& \times\left[\phi(1,2)-\phi_{r}(1,2)\right],
\end{aligned}
$$

where $F_{r}[\rho]$ is the intrinsic Helmholtz free energy of the reference system in which molecules interact via potential $\phi_{r}(1,2) . \mu$ is the chemical potential of the system. $v(\mathbf{r}, \hat{R})$ is the external potential, which arises from the ion-molecule interaction in our study of ion-induced nucleation. $g(1,2 ; \lambda)$ is the pair correlation function of the system with intermolecular potential,

$$
\phi_{\lambda}(1,2)=\phi_{r}(1,2)+\lambda\left[\phi(1,2)-\phi_{r}(1,2)\right] .
$$

We take a fluid of hard spheres as the reference system, for which $F_{r}[\rho]$ can be decomposed into the ideal gas contribution,

$$
F^{\text {ideal }}[\rho]=k_{B} T \int d 1 \rho(1)\left\{\log \left[\Lambda^{3} \rho(1)\right]-1\right\}
$$

and the excess free energy $F^{\mathrm{exc}}[\rho]$ due to the hard sphere exclusion. $k_{B}$ is the Boltzmann constant, $T$ is temperature, and $\Lambda$ is de Broglie's thermal wavelength. Following the previous work on homogeneous nucleation by Oxtoby and Evans,${ }^{16}$ we introduce the local density approximation, under which

$$
F^{\operatorname{exc}}[\rho] \approx \int d \mathbf{r}_{1} f^{\operatorname{exc}}\left(n\left(\mathbf{r}_{1}\right)\right) .
$$

To approximate the excess free energy density $f^{\text {exc }}(n(\mathbf{r}))$ per unit volume of the reference system of density $n$, CarnahanStarling's formula ${ }^{19}$ is employed

$$
f^{\mathrm{exc}}(n)=k_{B} \operatorname{Tn} \frac{y(4-3 y)}{(1-y)^{2}},
$$

where $y \equiv(\pi / 6) d^{3} n$. Because little is known about the paircorrelation functions of inhomogeneous fluids in which molecules assume orientational degrees of freedom, some approximation is required. Following Oxtoby and Evans, ${ }^{16}$ we employ the approximation

$$
g(1,2 ; \lambda) \approx H\left(r_{12}-d\right),
$$

where $H$ is the Heaviside step function and independent of $\lambda$. It follows that 


$$
\begin{aligned}
\Omega[\rho]= & k_{B} T \int d 1 \rho(1)\left\{\log \left[\Lambda^{3} \rho(1)\right]-1\right\} \\
& +\int d \mathbf{r}_{1} f^{\operatorname{exc}}\left(n\left(\mathbf{r}_{1}\right)\right)-\int d 1 \rho(1)[\mu-v(1)] \\
& +\frac{1}{2} \iint d 1 d 2 \rho(1) \rho(2) H\left(r_{12}-d\right) \\
& \times\left[\phi^{\text {att }}\left(r_{12}\right)+\phi^{p}(1,2)\right] .
\end{aligned}
$$

The equilibrium distribution for $\rho(\mathbf{r}, \hat{R})$ is determined by the stationarity condition of the grand potential

$$
\frac{\delta \Omega}{\delta \rho(1)}=0 \text {. }
$$

From Eqs. (16) and (17), we obtain

$$
\begin{aligned}
& k_{B} T \log \left[\Lambda^{3} \rho(1)\right]+\mu^{\mathrm{exc}}\left(n\left(\mathbf{r}_{1}\right)\right)-[\mu-v(1)] \\
& \quad+\int d 2 \rho(2) H\left(r_{12}-d\right)\left[\phi^{\mathrm{att}}\left(r_{12}\right)+\phi^{p}(1,2)\right]=0,
\end{aligned}
$$

where we have defined $\mu^{\mathrm{exc}}(n) \equiv \partial f^{\mathrm{exc}} / \partial n$. The solution of Eq. (18) can be used in Eq. (16) to evaluate $\Omega$ of the system.

In our study of ion-induced nucleation, an ion is taken as a point charge placed at the center of a hard sphere, which itself is supposed to be fixed at the center of a cluster. The ion-molecule interaction can be treated as an external potential, which is composed of the hard core repulsive potential and

$$
-p_{0} \hat{R} \cdot \mathbf{E}(\mathbf{u})
$$

where $\mathbf{E}$ is the electric field due to the ion. It will be readily observed from Eqs. (16), (18), and (19) that flipping the dipole moment embodied in each molecule and simultaneously reversing the direction of the electric field (by changing the sign of the ion charge) leaves $\rho(\mathbf{r}, \hat{R})$ and $\Omega$ unchanged. However, for a given direction of the dipole moments, both $\rho(\mathbf{r}, \hat{R})$ and $\Omega$ change when the sign of the ion charge is changed.

\section{B. Approximate formulas}

The solution of the full distribution function $\rho(\mathbf{r}, \hat{R})$ requires inordinate computer time and memory. To reduce the computational task, we make a further mean field approximation, where the orientational degrees of freedom $\hat{R}$ is replaced by an order parameter $\mathbf{p}(\mathbf{r})$ defined by

$$
\mathbf{p}(\mathbf{r}) \equiv \int d \hat{R} m(\mathbf{r}, \hat{R}) \hat{R} .
$$

As a result of interactions with other molecules and the external field, the molecule at $\mathbf{r}$ orients itself in various directions with a certain probability $m(\mathbf{r}, \hat{R})$. On average, however, the molecule can be described as having a dipole moment $\mathbf{p}(\mathbf{r})$. For a specified functional form of $\mathbf{p}(\mathbf{r})$, the distribution function $m(\mathbf{r}, \hat{R})$ consistent with this $\mathbf{p}(\mathbf{r})$ is not unique. Therefore, some approximation must be employed to introduce $\mathbf{p}(\mathbf{r})$ instead of $m(\mathbf{r}, \hat{R})$.

Without any loss of generality, Eq. (7) may be introduced into Eq. (16)

$$
\begin{aligned}
\Omega[n(\mathbf{r}), m(\mathbf{r}, \hat{R})]= & k_{B} T \int d 1 n\left(\mathbf{r}_{1}\right) m(1) \log m(1)+\int d \mathbf{r}_{1} f_{d}\left(n\left(\mathbf{r}_{1}\right)\right)-\int d 1 n\left(\mathbf{r}_{1}\right) m(1)[\mu-v(1)] \\
& +\frac{1}{2} \iint d \mathbf{r}_{1} d \mathbf{r}_{2} n\left(\mathbf{r}_{1}\right) n\left(\mathbf{r}_{2}\right) H\left(r_{12}-d\right) \phi^{\text {att }}\left(r_{12}\right)+\frac{1}{2} \iint d 1 d 2 n\left(\mathbf{r}_{1}\right) n\left(\mathbf{r}_{2}\right) \\
& \times m(1) m(2) H\left(r_{12}-d\right) \phi^{p}(1,2),
\end{aligned}
$$

where $f_{d}(n)$ is the Helmholtz free energy density per unit volume of the reference system, and is given by

$$
f_{d}(n)=k_{B} \operatorname{Tn}\left[\log \left(\Lambda^{3} n\right)-1\right]+f^{\mathrm{exc}}(n) .
$$

In the first term of Eq. (21), the quantity

$$
-k_{B} \int d \hat{R} m(\mathbf{r}, \hat{R}) \log m(\mathbf{r}, \hat{R})
$$

represents the entropy of a molecule at $\mathbf{r}$ associated with its orientational degrees of freedom. In order to express this quantity in terms of $\mathbf{p}(\mathbf{r})$, we choose a particular $m(\mathbf{r}, \hat{R})$ as an ansatz. In our mean field approximation, we take

$$
m(\mathbf{r}, \hat{R})=\frac{1}{Z} \exp \left(\frac{p_{0} \hat{R} \cdot \mathbf{E}_{0}}{k_{B} T}\right),
$$

the orientation distribution function of a molecule in an auxiliary uniform electric field $\mathbf{E}_{0} . Z$ is the normalization constant determined by Eq. (9)

$$
Z=\int d \hat{R} \exp \left(\frac{p_{0} \hat{R} \cdot \mathbf{E}_{0}}{k_{B} T}\right)=\frac{4 \pi k_{B} T}{p_{0} E_{0}} \sinh \left(\frac{p_{0} E_{0}}{k_{B} T}\right) .
$$

Namely, for every point $\mathbf{r}$ in the system, we assign $m(\mathbf{r}, \hat{R})$ which would be obtained if the molecule were subject to the uniform external field $\mathbf{E}_{0}$ and there were no interaction among molecules. The auxiliary electric field $\mathbf{E}_{0}$ to be imposed on the molecule is determined so that it yields the specified $\mathbf{p}(\mathbf{r})$ given by Eq. (20):

$$
p(\mathbf{r})=-\frac{1}{x}+\operatorname{coth} x
$$


where $x \equiv p_{0} E_{0} / k_{B} T$ and $\mathbf{p}(\mathbf{r})$ and $\mathbf{E}_{0}$ are parallel to each other. For the ansatz of $m(\mathbf{r}, \hat{R})$ employed here,

$$
-\int d \hat{R} m(\mathbf{r}, \hat{R}) \log m(\mathbf{r}, \hat{R})=\log \left(\frac{4 \pi}{x} \sinh x\right)-x p(\mathbf{r}),
$$

which, along with Eq. (26), serves as a parametric representation of the orientational entropy (divided by $k_{B}$ ) of a molecule. It is more convenient, however, to represent this quantity as a function of $\mathbf{p}(\mathbf{r})$ only. For this purpose, the righthand side of Eq. (26) and the logarithmic term in Eq. (27) are expanded in power series of $x$. Inverting the former series for $p(\mathbf{r})$ and substituting the resulting series in the latter, one finds

$$
\begin{aligned}
-\int & d \hat{R} m(\mathbf{r}, \hat{R}) \log m(\mathbf{r}, \hat{R}) \\
= & \log 4 \pi-\frac{3}{2} p(\mathbf{r})^{2}-\frac{9}{20} p(\mathbf{r})^{4}-\frac{99}{350} p(\mathbf{r})^{6} \\
& \quad-\frac{1539}{7000} p(\mathbf{r})^{8}+O\left(p^{10}\right) .
\end{aligned}
$$

From the parametric representation Eqs. (26) and (27), it can be shown that

$-\int d \hat{R} m(\mathbf{r}, \hat{R}) \log m(\mathbf{r}, \hat{R}) \sim \log \left[1-p(\mathbf{r})^{2}\right]$

as $p(\mathbf{r})^{2} \rightarrow 1$.

To recapture such limiting behavior, we employ the Padé approximation, by which the right-hand side of Eq. (28) is resummed as

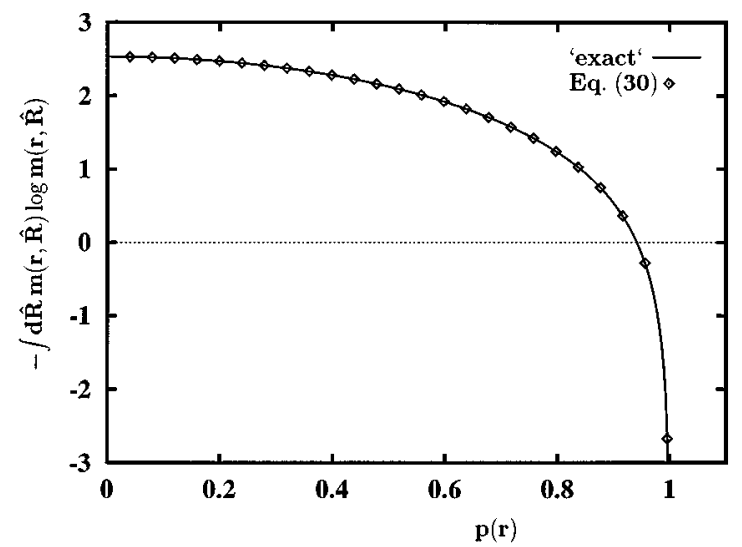

FIG. 2. Orientational entropy per molecule (divided by $k_{B}$ ). The Padé approximation, Eq. (30), is compared with the "exact" result using the parametric representation, Eqs. (26) and (27).

$$
\begin{aligned}
-\int & d \hat{R} m(\mathbf{r}, \hat{R}) \log m(\mathbf{r}, \hat{R}) \\
\approx & \log 4 \pi+\log \left[1-p(\mathbf{r})^{2}\right] \\
& \quad-\frac{1}{2} p(\mathbf{r})^{2}+\frac{1}{20} p(\mathbf{r})^{4}+\frac{53}{1050} p(\mathbf{r})^{6}+\frac{211}{7000} p(\mathbf{r})^{8} \\
\equiv & s^{\operatorname{ort}}(p(\mathbf{r})),
\end{aligned}
$$

where the coefficients of $p(\mathbf{r})^{2 k}$ are determined so as to ensure the matching of two expressions at the small $p(\mathbf{r})$ limit. In Fig. 2, Eq. (30) is compared with the "exact" result using parametric representation given by Eqs. (26) and (27). As is seen from Fig. 2, the two representations are indistinguishable.

The remainder of the terms in Eq. (21) can be treated similarly. To further simplify the equation, we have expanded $v(1)$ and $\phi^{p}(1,2)$ around $a=0$. Retaining the terms up to $O(a / d)$, we obtain

$$
\begin{aligned}
\Omega[n(\mathbf{r}), \mathbf{p}(\mathbf{r})]= & -k_{B} T \int d \mathbf{r}_{1} n\left(\mathbf{r}_{1}\right) s^{\mathrm{ort}}\left(p\left(\mathbf{r}_{1}\right)\right)+\int d \mathbf{r}_{1} f_{d}\left(n\left(\mathbf{r}_{1}\right)\right)-\int d \mathbf{r}_{1} n\left(\mathbf{r}_{1}\right)\left[\mu+p_{0} p_{\alpha}\left(\mathbf{r}_{1}\right)\left(E_{\alpha}\left(\mathbf{r}_{1}\right)\right.\right. \\
& \left.\left.+\left.\frac{3}{5} a \frac{\partial E_{\alpha}}{\partial x_{\beta}}\right|_{\mathbf{r}=\mathbf{r}_{1}} p_{\beta}\left(\mathbf{r}_{1}\right)\right)\right]+\frac{1}{2} \iint d \mathbf{r}_{1} d \mathbf{r}_{2} n\left(\mathbf{r}_{1}\right) n\left(\mathbf{r}_{2}\right) H\left(r_{12}-d\right) \phi^{\text {att }}\left(r_{12}\right) \\
& +\frac{1}{2} \iint d \mathbf{r}_{1} d \mathbf{r}_{2} n\left(\mathbf{r}_{1}\right) n\left(\mathbf{r}_{2}\right) H\left(r_{12}-d\right)\left(\Phi_{\alpha \beta}^{p}\left(\mathbf{r}_{12}\right)+\left.\frac{3}{5} a \frac{\partial \Phi_{\alpha \beta}^{p}}{\partial x_{\gamma}}\right|_{\mathbf{r}=\mathbf{r}_{12}}\left[p_{\gamma}\left(\mathbf{r}_{2}\right)-p_{\gamma}\left(\mathbf{r}_{1}\right)\right]\right) p_{\alpha}\left(\mathbf{r}_{1}\right) p_{\beta}\left(\mathbf{r}_{2}\right),
\end{aligned}
$$

where, for clarity, we use tensor notation to represent electrostatic terms. $\Phi_{\alpha \beta}^{p}(\mathbf{r})$ is defined by

$$
\Phi_{\alpha \beta}^{p}(\mathbf{r})=p_{0}^{2}\left(\frac{\delta_{\alpha \beta}}{r^{3}}-\frac{3 x_{\alpha} x_{\beta}}{r^{5}}\right),
$$

and $x_{\alpha}$ is the $\alpha$ th component of $\mathbf{r}$ in Cartesian coordinate system. In arriving at Eq. (31) we have used the following relation:

$$
\int d \hat{R} m(\mathbf{r}, \hat{R}) \hat{R}_{\alpha} \hat{R}_{\beta}=\left(\frac{1}{3}-\frac{1}{5} p(\mathbf{r})^{2}\right) \delta_{\alpha \beta}+\frac{3}{5} p_{\alpha}(\mathbf{r}) p_{\beta}(\mathbf{r})+O\left(p^{4}\right)
$$

by employing the ansatz for $m(\mathbf{r}, \hat{R})$ given by Eq. (24). As before, the stationarity condition of $\Omega$ determines the equilibrium profiles for $n(\mathbf{r})$ and $\mathbf{p}(\mathbf{r})$ : 


$$
\frac{\delta \Omega}{\delta n\left(\mathbf{r}_{1}\right)}=0, \quad \frac{\delta \Omega}{\delta \mathbf{p}\left(\mathbf{r}_{1}\right)}=0,
$$

subject to boundary conditions imposed by the hard core interaction with an ion. Written explicitly,

$$
\begin{aligned}
0= & -k_{B} T S^{\mathrm{ort}}\left[p\left(\mathbf{r}_{1}\right)\right]+\mu_{d}\left[n\left(\mathbf{r}_{1}\right)\right]-\left[\mu+p_{0} p_{\alpha}\left(\mathbf{r}_{1}\right)\left(E_{\alpha}\left(\mathbf{r}_{1}\right)+\left.\frac{3}{5} a \frac{\partial E_{\alpha}}{\partial x_{\beta}}\right|_{\mathbf{r}=\mathbf{r}_{1}} p_{\beta}\left(\mathbf{r}_{1}\right)\right)\right]+\int d \mathbf{r}_{2} n\left(\mathbf{r}_{2}\right) H\left(r_{12}-d\right) \phi^{\text {att }}\left(r_{12}\right) \\
& +\int d \mathbf{r}_{2} n\left(\mathbf{r}_{2}\right) H\left(r_{12}-d\right)\left(\Phi_{\alpha \beta}^{p}\left(\mathbf{r}_{12}\right)+\left.\frac{3}{5} a \frac{\partial \Phi_{\alpha \beta}^{p}}{\partial x_{\gamma}}\right|_{\mathbf{r}=\mathbf{r}_{12}}\left[p_{\gamma}\left(\mathbf{r}_{2}\right)-p_{\gamma}\left(\mathbf{r}_{1}\right)\right]\right) p_{\alpha}\left(\mathbf{r}_{1}\right) p_{\beta}\left(\mathbf{r}_{2}\right)
\end{aligned}
$$

and

$$
\begin{aligned}
0= & -k_{B} T \frac{d s^{\mathrm{ort}}}{d p\left(\mathbf{r}_{1}\right)} \frac{p_{\alpha}\left(\mathbf{r}_{1}\right)}{p\left(\mathbf{r}_{1}\right)}-p_{0}\left(E_{\alpha}\left(\mathbf{r}_{1}\right)+\left.\frac{6}{5} a \frac{\partial E_{\alpha}}{\partial x_{\beta}}\right|_{\mathbf{r}=\mathbf{r}_{1}} p_{\beta}\left(\mathbf{r}_{1}\right)\right)+\int d \mathbf{r}_{2} n\left(\mathbf{r}_{2}\right) H\left(r_{12}-d\right)\left(\Phi_{\alpha \beta}^{p}\left(\mathbf{r}_{12}\right)\right. \\
& \left.+\left.\frac{3}{5} a \frac{\partial \Phi_{\alpha \beta}^{p}}{\partial x_{\gamma}}\right|_{\mathbf{r}=\mathbf{r}_{12}}\left[p_{\gamma}\left(\mathbf{r}_{2}\right)-2 p_{\gamma}\left(\mathbf{r}_{1}\right)\right]\right) p_{\beta}\left(\mathbf{r}_{2}\right),
\end{aligned}
$$

where use has been made of the fact that both $\partial E_{\alpha} / \partial x_{\beta}$ and $\partial \Phi_{\alpha \beta}^{p} / \partial x_{\gamma}$ are invariant with respect to any exchange of indices and that $\Phi_{\alpha \beta}^{p}(-\mathbf{r})=\Phi_{\alpha \beta}^{p}(\mathbf{r})$ while $\partial \Phi_{\alpha \beta}^{p} /\left.\partial x_{\gamma}\right|_{\mathbf{r}=\mathbf{r}_{12}}$ $=-\partial \Phi_{\alpha \beta}^{p} /\left.\partial x_{\gamma}\right|_{\mathbf{r}=\mathbf{r}_{21}}$. We have defined $\mu_{d}(n) \equiv \partial f_{d} / \partial n$.

In our study of ion-induced nucleation, the system is spherically symmetric about the center of an ion. Taking this as the origin, the electric field is given by

$$
E_{\alpha}(\mathbf{r})=q \frac{x_{\alpha}}{r^{3}},
$$

where $q$ is the ion charge. For the particular choice of $\phi^{\text {att }}\left(r_{12}\right)$ in Eq. (3), integrations with respect to the polar and azimuthal angles in Eqs. (31), (35), and (36) become analytically tractable, reducing the dimensionality of the integrals to two for Eq. (31), and one for Eqs. (35) and (36). We may now discretize the domain of $\mathbf{r}$. It can be shown that the terms arising from the dipole-dipole interaction become identically zero unless $r_{1}$ and $r_{2}$ satisfy

$$
\begin{aligned}
\left(r_{1}, r_{2}\right) \in & \left\{r_{\text {ion }}+d / 2 \leqslant r_{1} \leqslant r_{\text {ion }}+3 d / 2\right. \\
& \text { and } \left.r_{\text {ion }}+d / 2 \leqslant r_{2} \leqslant r_{1}+d\right\} \\
& \text { or }\left\{r_{\text {ion }}+3 d / 2 \leqslant r_{1} \leqslant r_{0}\right. \\
& \text { and } \left.r_{1}-d \leqslant r_{2} \leqslant r_{1}+d\right\},
\end{aligned}
$$

where $r_{0}$ is the radius of the system boundary. The condition (38) results from the volume exclusion represented by $H\left(r_{12}-d\right)$. The contribution due to the dipole-dipole interaction is then expected to be small, and neglected as a first approximation. The validity of this approximation will be examined in Sec. IV. The grand potential now becomes

$$
\begin{aligned}
\Omega[n(r), p(r)]= & -4 \pi k_{B} T \int r_{1}^{2} d r_{1} n\left(r_{1}\right) s^{\mathrm{ort}}\left(p\left(r_{1}\right)\right)+4 \pi \int r_{1}^{2} d r_{1} f_{d}\left(n\left(r_{1}\right)\right)-4 \pi \int r_{1}^{2} d r_{1} n\left(r_{1}\right) \\
& \times\left[\mu+p_{0} q \frac{p\left(r_{1}\right)}{r_{1}^{2}}\left(1-\frac{6}{5} \frac{a}{r_{1}} p\left(r_{1}\right)\right)\right]+4 \pi \int r_{1}^{2} d r_{1} n\left(r_{1}\right)\left(\pi \int r_{2}^{2} d r_{2} n\left(r_{2}\right)\right. \\
& \left.\times \int_{-1}^{c\left(r_{1}, r_{2}\right)} d(\cos \theta) \phi^{\mathrm{att}}\left(r_{12}\right)\right) .
\end{aligned}
$$

Note that

$$
\begin{aligned}
\phi^{\text {att }}\left(r_{12}\right)= & \epsilon d^{6} \frac{d}{d(\cos \theta)}\left(-\frac{1}{4 r_{1} r_{2}}\right. \\
& \left.\times \frac{1}{\left(r_{1}^{2}+r_{2}^{2}-2 r_{1} r_{2} \cos \theta\right)^{2}}\right) .
\end{aligned}
$$

$c\left(r_{1}, r_{2}\right)$ is unity except when $r_{1}$ and $r_{2}$ satisfy the condition (38), in which case

$$
c\left(r_{1}, r_{2}\right)=\frac{r_{1}^{2}+r_{2}^{2}-d^{2}}{2 r_{1} r_{2}} .
$$

The sign preference of the grand potential is clearly seen in Eq. (39). Consider a system in equilibrium and suppose that the sign of the charge $q$ is reversed. If $a$ were zero, the 
system would establish a new equilibrium state by simply reversing the sign of $p(r)$ without changing its magnitude or $n(r)$, since the grand potential is invariant with respect to simultaneous reversal of $q$ and $p(r)$. However, for any nonzero value of $a, \Omega$ is no longer invariant and the system has to modify both $n(r)$ and the magnitude of $p(r)$ as well as its sign to establish a new equilibrium state, which in general affects the value of $\Omega$. The equilibrium profiles for $n(r)$ and $p(r)$ are determined by

$$
\begin{aligned}
0= & -k_{B} T s^{\text {ort }}\left(p\left(r_{1}\right)\right)+\mu_{d}\left(n\left(r_{1}\right)\right)-\left[\mu+p_{0} q \frac{p\left(r_{1}\right)}{r_{1}^{2}}\right. \\
& \left.\times\left(1-\frac{6}{5} \frac{a}{r_{1}} p\left(r_{1}\right)\right)\right]+2 \pi \int r_{2}^{2} d r_{2} n\left(r_{2}\right) \\
& \times \int_{-1}^{c\left(r_{1}, r_{2}\right)} d(\cos \theta) \phi^{\text {att }}\left(r_{12}\right)
\end{aligned}
$$

and

$$
0=-k_{B} T \frac{d s^{\text {ort }}}{d p\left(r_{1}\right)}-\frac{p_{0} q}{r_{1}^{2}}\left(1-\frac{12}{5} \frac{a}{r_{1}} p\left(r_{1}\right)\right) .
$$

Note that Eq. (43) is now independent of $n(r)$ as a result of ignoring the dipole-dipole interaction.

\section{Bulk properties}

In the absence of the external field, the thermodynamic properties of a homogeneous system can be easily derived from Eq. (16). Since such a system is isotropic as well, we may set $\rho(\mathbf{r}, \hat{R})=n / 4 \pi$ in Eq. (16), from which the Helmholtz free energy density per unit volume of the system of density $n$ is obtained:

$$
f(T, n)=-p+\mu n=-k_{B} T n \log 4 \pi+f_{d}(n)-\frac{\alpha}{8 \pi} n^{2} .
$$

Note that $\Omega=-p V$ for a homogeneous system. $\alpha$ is defined here by

$$
\begin{aligned}
\alpha & \equiv-\frac{1}{4 \pi V} \iint d 1 d 2 H\left(r_{12}-d\right)\left[\phi^{\mathrm{att}}\left(r_{12}\right)+\phi^{p}(1,2)\right] \\
& =-4 \pi \int d \mathbf{r}_{12} H\left(r_{12}-d\right) \phi^{\mathrm{att}}\left(r_{12}\right),
\end{aligned}
$$

where we have used the fact that the integral of $\phi^{p}(1,2)$ is zero. For the particular choice of $\phi^{\text {att }}\left(r_{12}\right)$ given by Eq. (3), one has

$$
\alpha=\frac{(4 \pi)^{2}}{3} \epsilon d^{3} .
$$

Equation (44) is a fundamental equation of the isotropic system, from which by well known thermodynamic relations, one obtains

$$
\begin{aligned}
& \mu(T, n)=-k_{B} T \log 4 \pi+\mu_{d}(n)-\frac{\alpha}{4 \pi} n ; \\
& p(T, n)=n \mu_{d}(n)-f_{d}(T, n)-\frac{\alpha}{8 \pi} n^{2} .
\end{aligned}
$$

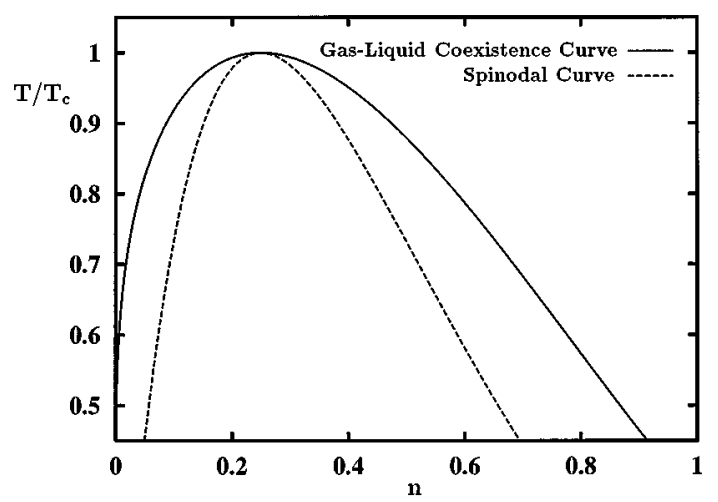

FIG. 3. The gas-liquid coexistence curve and the spinodal curve calculated for the system composed of the dipole molecules. Density is normalized by $d^{-3}$.

Equation (47) is identical with Eq. (18) applied to the isotropic system at $v(\mathbf{r}, \hat{R})=0$. These results could also have been obtained by setting $\mathbf{E}=\mathbf{0}$ and $\mathbf{p}=\mathbf{0}$ in Eq. (31).

At a given temperature, the coexisting bulk densities are determined by

$$
\begin{aligned}
& \mu_{l}\left(T, n_{l}^{\mathrm{eq}}\right)=\mu_{v}\left(T, n_{v}^{\mathrm{eq}}\right) ; \\
& p_{l}\left(T, n_{l}^{\mathrm{eq}}\right)=p_{v}\left(T, n_{v}^{\mathrm{eq}}\right),
\end{aligned}
$$

where the subscripts $l$ and $v$ refer to liquid and vapor, respectively. The spinodal curve which divides the metastable and unstable regions in $T-n$ phase diagram is obtained by

$$
\frac{\partial p}{\partial n}=0 \text {. }
$$

The critical point is located in the phase diagram by Eq. (50) and

$$
\frac{\partial^{2} p}{\partial n^{2}}=0
$$

with a numerical solution

$$
\begin{aligned}
& y_{c}=\frac{\pi}{6} d^{3} n_{c} \approx 0.13044 ; \\
& \frac{1}{k_{B} T_{c}} \approx 14.135\left(\frac{\pi^{2} d^{3}}{\alpha}\right)=2.6503\left(\frac{1}{\epsilon}\right) .
\end{aligned}
$$

The $T-n$ phase diagram is shown in Fig. 3 .

\section{SOLUTION METHOD FOR THE EQUILIBRIUM PROFILES}

Given $T / T_{c}$ and supersaturation $S$, defined as the ratio of the metastable vapor pressure and the equilibrium vapor pressure, we can calculate, via Eqs. (47)-(49), the chemical potential $\mu$ of the system and the densities of the bulk liquid $n_{l}$ and vapor $n_{v}$ at that chemical potential. As mentioned in Sec. II B, Eq. (43) is independent of $n(r)$ and can be easily solved numerically. The solution is substituted in Eq. (42), which is now an integral equation for $n(r)$ only and can be solved by iteration. In the case of homogeneous nucleation, 
the equation to determine the density profile $n(r)$ has two solutions. One is that of a metastable vapor, namely $n(r)=n_{v}$. The other is that of a critical nucleus, which corresponds to a saddle point in functional space. When an ion is present in the vapor, the metastable profile exhibits solvation of the ion. This metastable nucleus was obtained by starting from the initial guess $n(r)=n_{v}$. Iteration can be repeated until the quantity

$$
\Delta \equiv \int d \mathbf{r}\left|\frac{\delta \Omega}{\delta n(\mathbf{r})}\right|
$$

becomes sufficiently small. The iteration process applicable to a critical nucleus is described in detail by Oxtoby and Evans. ${ }^{16}$ As the initial guess, we take the step function:

$$
n(r)=\left\{\begin{array}{lc}
n_{l}, & r \leqslant R \\
n_{v}, & \text { otherwise }
\end{array} .\right.
$$

If $R$ is too small, the nucleus shrinks as the iteration proceeds, while it grows if $R$ is too large. Starting from several values of $R$, it is possible to find $R^{*}$ such that the nucleus neither shrinks nor grows as the iteration is repeated. Our solution method differs from that proposed by Oxtoby and Evans ${ }^{16}$ in some details. Instead of monitoring the behavior of $\Omega$ as a function of iteration number, we monitored $\Delta$. $R^{*}$ was identified with that which yields, after some steps of iteration, $n(r)$ that minimizes $\Delta$. Then, this $n(r)$ was used as the initial guess in solving Eq. (42) more accurately by the Newton-Raphson method, which was repeatedly applied until $\Delta$ becomes sufficiently small.

Finally, the boundary of the system is taken to be a sphere, the radius of which is sufficiently large so that $n(r)$ attains its limiting value $n_{v}$ there. As a results of the long ranged nature of the monopole-dipole interaction, $p(r)$ may not reach its limiting value of zero even at $r_{0}$. The truncation error thus introduced, however, exactly cancels out when the difference is taken, as in the case of the reversible work calculation, between two states with the same value of $n_{v}$.

\section{RESULTS AND THEIR IMPLICATIONS}

It is advantageous to normalize the equations by model parameters: $d$ for the length scale; $k_{B} T_{c}$ for the energy scale. When the dipole-dipole interaction is ignored, the dipole moment $p_{0}$ and the charge $q$ of an ion can be lumped together to form a single dimensionless parameter

$$
\chi \equiv \frac{p_{0} q}{d^{2} k_{B} T_{c}},
$$

which is the ratio between the monopole-dipole interaction energy at separation $d$, the diameter of a molecule, and the thermal energy at the bulk critical point. $\chi>0$ corresponds to nucleation either around a positive ion of molecules each with dipole pointing outward from its center, or around a negative ion of molecules each with dipole pointing toward its center.

Typical values of $\chi$ are of the order of 10 . When $p_{0}=1$ $\mathrm{D}$ (Debye), $d=3 \AA, T_{c}=10^{3} \mathrm{~K}$, and $q=e$, where $-e$ is the charge of an electron, $\chi \approx 3.9$. Our choice of parameters is

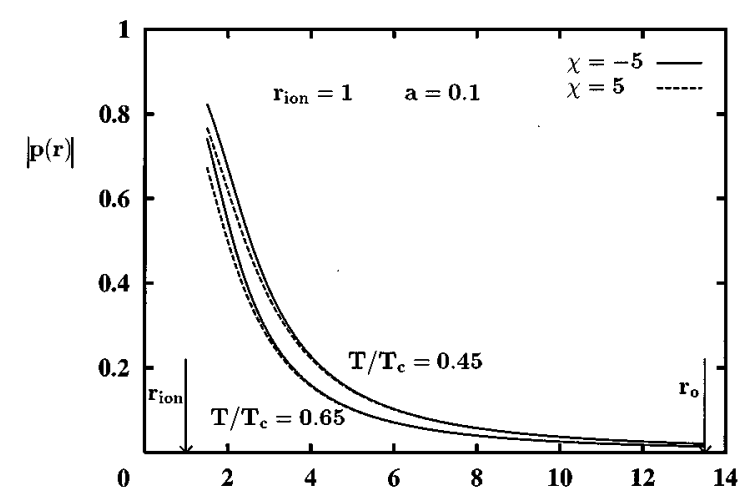

FIG. 4. The magnitude of an average dipole moment distribution at two different temperatures. $r_{0}$ is the radius of the system boundary. Distance is normalized by $d$.

$|\chi|=5, a=0.1 d$, and the ionic radius $r_{\text {ion }}=d$. Later, the effect of the values of these parameters on the reversible work of nucleation will be examined.

The absolute value of the average dipole moment distribution $p(r)$ is shown in Fig. 4. The sign of $p(r)$ is the same as that of $\chi$. Near the ion, dipoles tend to line up with the electric field, showing $r^{-2}$ decay for larger distance, as it should. What is not shown in Fig. 4 is the case of $\chi=0$, when Eq. (43) has a trivial solution $p(r) \equiv 0$. Figure 4 shows that at a given distance $r$ from the ion, the magnitude of the average dipole moment is always larger when $\chi<0$. This is readily understood as follows. Suppose for a moment that the dipole moment is placed outward with respect to the center of each molecule. The dipole moment in a molecule at $r$ tends to point toward a negative ion, thereby decreasing its distance from the ion, while it will point away from a positive ion, increasing its distance from the ion. For a given value of $|\chi|$ and at the fixed ion-molecule distance, the monopole-dipole interaction is therefore stronger when $\chi<0$ than when $\chi>0$. As seen from Eq. (43), $T / T_{c}$ affects $p(r)$ through the parameter $\chi\left(T / T_{c}\right)^{-1}$. Increasing $T / T_{c}$ has the same effect as decreasing $\chi$, as shown in Fig. 4. Stated differently, the entropic contribution becomes more important at higher $T / T_{c}$, and the system prefers a less ordered state.

The number density distribution $n(r)$ is obtained from Eq. (42). Figure 5(a) shows the solution at $T / T_{c}=0.65$ and supersaturation $S=3$. Density profile for the case of $\chi<0$ is shown in Fig. 5(b) at the same temperature for several values of supersaturation. Figures 5(c) and 5(d), show similar results at $T / T_{c}=0.45$.

As mentioned in Sec. III, a metastable profile shows solvation of the ion. The exception is the case of $\chi=0$, corresponding to nucleation on a neutral particle, for which the profile shows a decrease in density near the particle. This volume exclusion effect is a result of the repulsive hard core interaction between the particle of radius $r_{\text {ion }}$ and the molecules. Although the effect exists for other cases as well, it is often overwhelmed by the attractive interaction between the monopole and the dipoles. The general feature to be observed is that at a given supersaturation $S$, the metastable nucleus with $\chi<0$ is always larger than that with $\chi>0$, while 


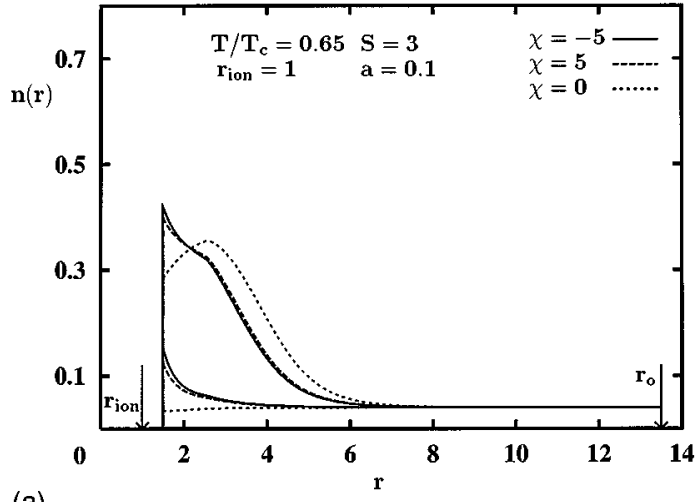

(a)

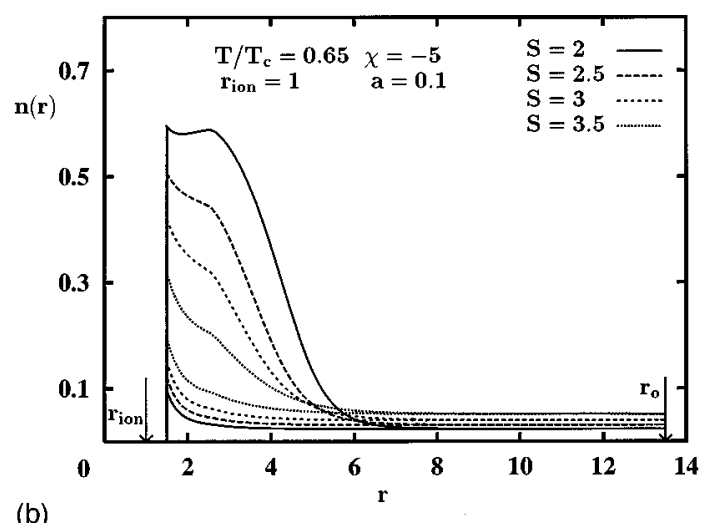

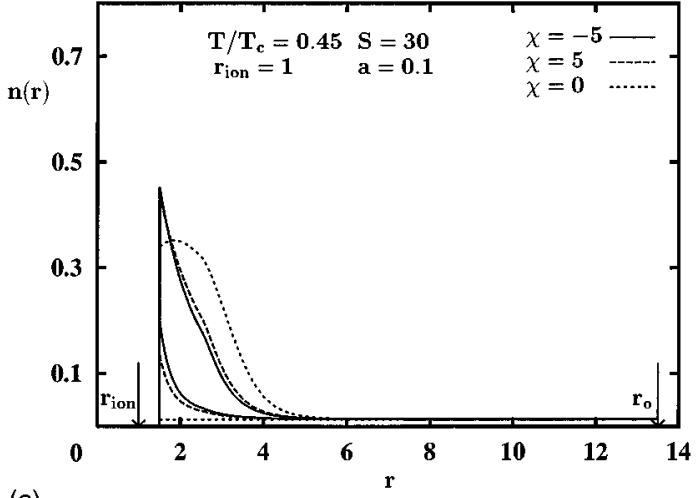

(c)

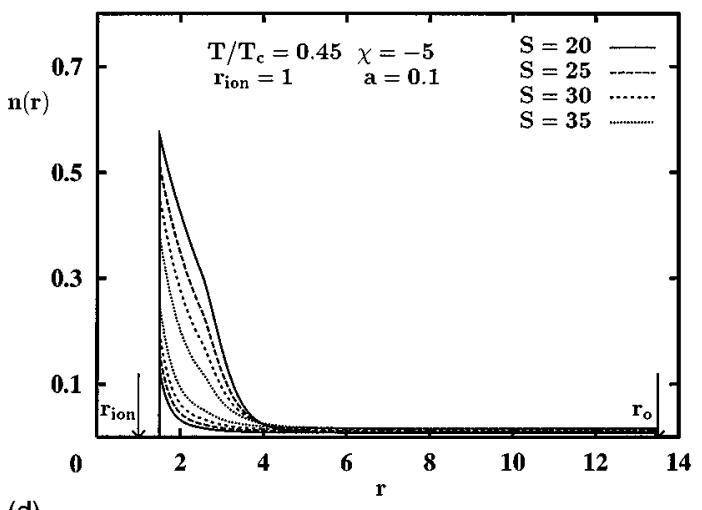

(d)

FIG. 5. (a) Equilibrium density profiles at $T / T_{c}=0.65$ and $S=3$. The profiles with smaller values of $n(r)$ correspond to the metastable nuclei, while those with a larger values of $n(r)$ represent the critical nuclei. $r_{0}$ is the radius of the system boundary. Distance is normalized by $d$. (b) Variation of the density profile with the supersaturation $S . T / T_{c}=0.65$. (c) Same as (a), but for $T / T_{c}=0.45$ and $S=30$. (d) Same as (b), but for $T / T_{c}=0.45$.

the opposite is true for the critical nuclei. Also, the metastable nucleus grows as $S$ increases, while the critical nucleus shrinks. To qualitatively understand such features, it is helpful to take a molecular point of view. An equilibrium nucleus is then characterized as the one for which the rate of evaporation of molecules (per unit time unit area) and that of condensation is balanced. The qualitative behavior of these rates is shown schematically in Fig. 6, where we denote by $N$

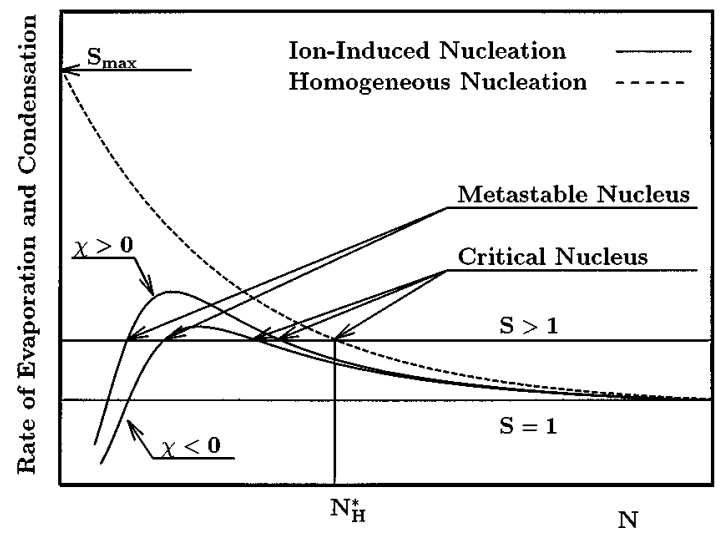

FIG. 6. A schematic picture of the rate of evaporation (represented by the curves) and condensation (represented by the horizontal lines) of molecules per unit area of the nucleus. The abscissa represents the number of molecules in the nucleus. the number of molecules within a nucleus. At a given temperature, the physical state of the nucleus and therefore the rate of evaporation are independent of the vapor pressure. While $N$ is relatively small, the intermolecular interaction energy per molecule increases (in its magnitude) as $N$ increases. Thus the rate is a monotonically decreasing function of $N$ and approaches the bulk liquid value equal to the condensation rate at saturation $(S=1)$ as $N$ approaches infinity. The condensation rate, on the other hand, is independent of $N$ and is proportional to the vapor pressure or $S$. As shown in Fig. 6, those two rates are equal when $N=N_{H}^{*}$, and $N_{H}^{*}$ decreases as $S$ increases, approaching order of one at the spinodal (denoted by $S_{\max }$ ), where an infinitesimal fluctuation in density promotes phase transition.

When an ion is introduced in a nucleus, it attracts molecules via the monopole-dipole interaction, thereby decreasing the evaporation rate. Since the electric field decays as $r^{-2}$, the decrease should be most significant for small $N$ and becomes negligible as $N$ approaches infinity. As mentioned in connection with Fig. 4, this attraction is stronger when $\chi<0$ than when $\chi>0$, which yields the curves for $\chi>0$ and $\chi<0$ in Fig. 6. When the attraction between an ion and molecules is sufficiently large, the condensation rate balances the evaporation rate even when $S \leqslant 1$, which corresponds to vapor solvation of the ion. From Fig. 6 , it is clear that at a given supersaturation $S$, a metastable nucleus is larger if $\chi<0$, while the opposite is true for a critical nucleus. Figure 


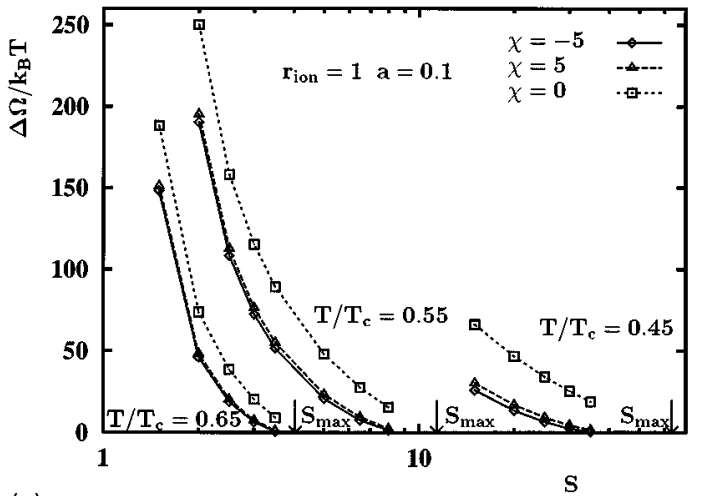

(a)

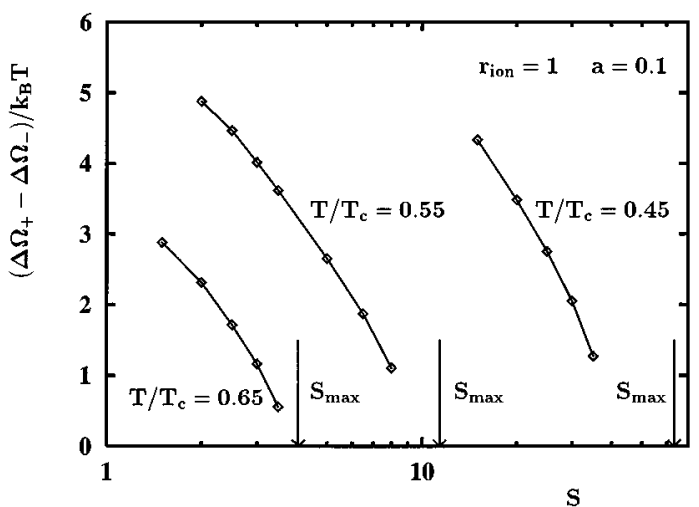

(b)

FIG. 7. (a) The reversible work of nucleation. Three values of $S_{\max }$ represent the supersaturation at the stability limit. From the left, they correspond to the value at $T / T_{c}=0.65$, at $T / T_{c}=0.55$, and at $T / T_{c}=0.45$, respectively. (b) The difference in the reversible work reported in (a) between the case of $\chi>0$ and that with $\chi<0$.

6 also shows that as $S$ increases, a metastable nucleus grows while a critical nucleus shrinks, and that two nuclei coincide, meaning that the system reaches its stability limit, at the supersaturation lower than $S_{\max }$. A similar figure is employed by Castleman et al. ${ }^{20}$ in explaining the existence of a metastable nucleus and the instability of the system at a supersaturation lower than $S_{\max }$.

Figure 7(a) shows the reversible work $\Delta \Omega / k_{B} T$ of nucleation as a function of supersaturation. The reversible work relevant here is that is required to form a critical nucleus from the metastable one. Figure 7 shows that the reversible work decreases as $S$ is increased, and that if $\chi \neq 0$ it approaches zero before $S_{\max }$ is reached, which is consistent with the qualitative prediction of Fig. 6. Figure 7(b) shows the difference between the reversible work $\Delta \Omega_{+} / k_{B} T$ of nucleation with $\chi>0$ and that $\left(\Delta \Omega_{-} / k_{B} T\right)$ with $\chi<0$. Figure 7(b) clearly shows the preference for the case of $\chi<0$, influencing the nucleation rate by a factor of $10-10^{2}$. The observed preference can be understood along the line of discussion given in connection with the average dipole distribution $p(r)$. Figure 7(b) also shows a monotonic decrease in $\Delta \Omega_{+}-\Delta \Omega_{-}$with increasing $S$. To understand its implication, let us rearrange this quantity as follows:

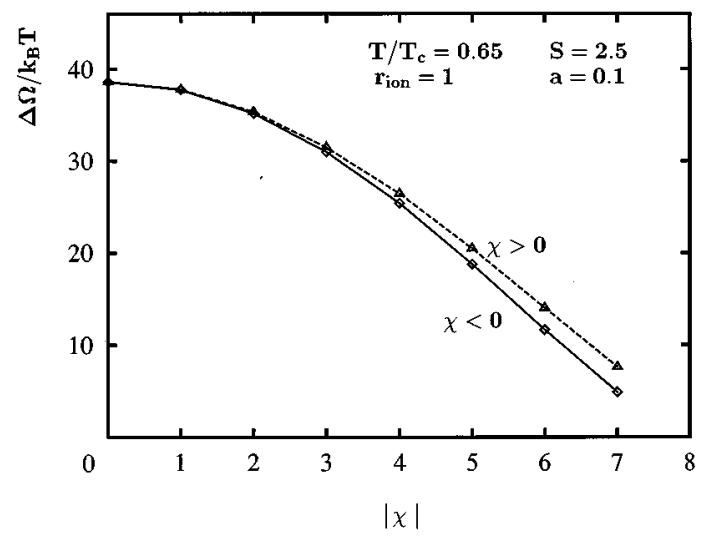

FIG. 8. Effect of the value of $\chi$ on the reversible work.

$$
\begin{aligned}
\Delta \Omega_{+}-\Delta \Omega_{-}= & \left(\Omega_{+}^{\text {critical }}-\Omega_{-}^{\text {critical }}\right) \\
& -\left(\Omega_{+}^{\text {metastable }}-\Omega_{-}^{\text {metastable }}\right) .
\end{aligned}
$$

For an equilibrium distribution of $p(r)$ and $n(r)$, one may combine Eq. (42) with Eq. (39) to eliminate the double integral. Expanding the resulting expression for $\Omega_{+}$around $\bar{n} \equiv\left(n_{+}+n_{-}\right) / 2$ and $\bar{p} \equiv\left(p_{+}-p_{-}\right) / 2$, and that for $\Omega_{-}$ around $\bar{n}$ and $-\bar{p}$, one finds

$$
\begin{aligned}
\Omega_{+}-\Omega_{-} \approx & \frac{6}{5} a\left|p_{0} q\right| \int d \mathbf{r} \frac{\bar{n} \bar{p}^{2}}{r^{3}}+\int d \mathbf{r}\left[h_{1}(\bar{n}, \bar{p})\right. \\
& \left.\times\left(n_{+}-n_{-}\right)+\bar{n} h_{2}(\bar{p})\left(p_{+}+p_{-}\right)\right],
\end{aligned}
$$

where $h_{1}$ and $h_{2}$ are certain functions. Noting that $p(r)$ is independent of $n(r)$ and retaining only the leading term,

$$
\Delta \Omega_{+}-\Delta \Omega_{-} \approx \frac{6}{5} a\left|p_{0} q\right| \int d \mathbf{r} \frac{\bar{p}^{2}}{r^{3}}\left(\bar{n}^{\text {critical }}-\bar{n}^{\text {metastable }}\right) .
$$

It is clear that this quantity decreases monotonically as $S$ is increased.

The dependence of the reversible work on the values of model parameters is shown in Figs. 8-10. Figure 8 shows that the presence of the electric field always decreases the reversible work of nucleation of dipolar molecules. Figure 9

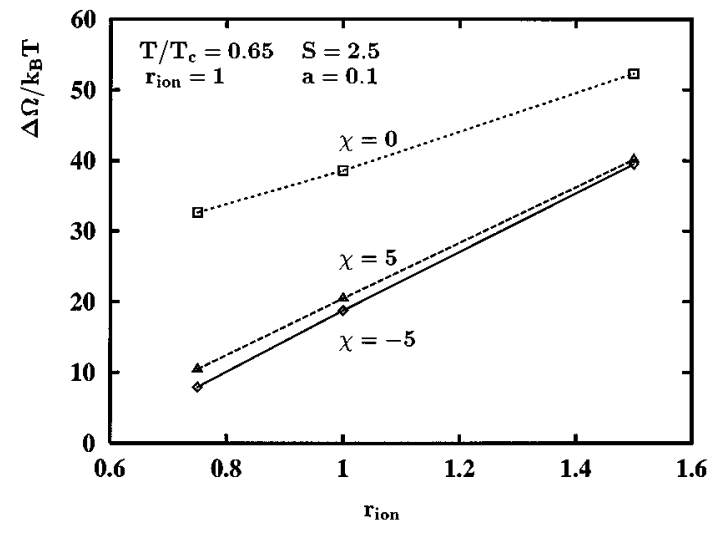

FIG. 9. Dependence of the reversible work on the size of an ion. Distance is normalized by $d$. 


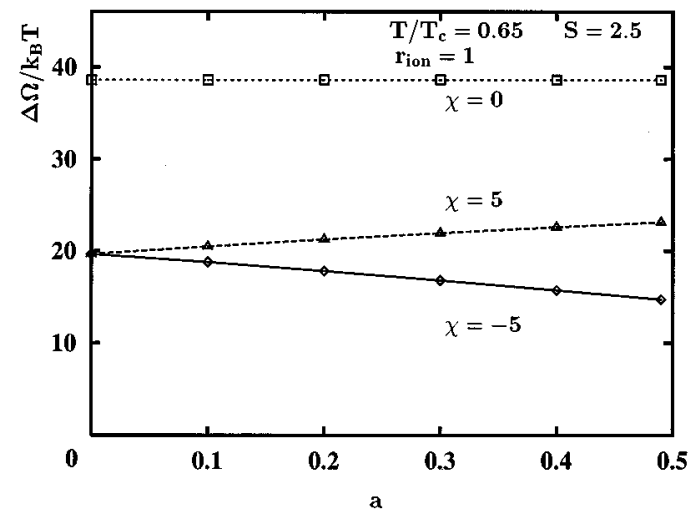

FIG. 10. Effect of asymmetry of a molecule upon the sign preference of the reversible work of ion-induced nucleation. Distance is normalized by $d$.

shows that the increase in $r_{\text {ion }}$ results in the increase of the reversible work as well as in the decrease in sign dependence. This is expected since the increase in $r_{\text {ion }}$ causes the increase in the monopole-dipole separation. However, as seen from Fig. 10, it is the asymmetric nature of molecules that is directly responsible for the sign dependence of the reversible work. Figure 10 shows that the reversible work for the case of $\chi>0$ increases as $a$ is increased, while it decreases if $\chi<0$. Such a trend can be readily understood by noting that a larger value of $a$ implies larger monopoledipole separation if $\chi>0$ and smaller separation if $\chi<0$ for a given ion-molecule separation. Equation (26) shows that, in the weak electric field limit, $p \sim p_{0} q$. From Eqs. (55) and (58), one finds

$$
\Delta \Omega_{+}-\Delta \Omega_{-} \sim a|\chi|^{3} .
$$

Such dependence on $|\chi|$ and $a$ is clearly seen in Figs. 11(a) and 11(b).

In our calculation, the dipole-dipole interaction has been ignored for computational convenience. To examine the validity of this approximation, we calculated its contribution to the grand potential of the system. As a self-consistency check, we use the equilibrium profile $p(r)$ and $n(r)$ obtained by neglecting the dipole-dipole interaction. In Eq. (31), when the integrations with respect to $\mathbf{r}_{2}$ are carried out,

$$
\omega^{\text {att }}\left(\mathbf{r}_{1}\right)=\frac{1}{2} \int d \mathbf{r}_{2} n\left(\mathbf{r}_{2}\right) H\left(r_{12}-d\right) \phi^{\text {att }}\left(r_{12}\right)
$$

and

$$
\begin{aligned}
\omega^{p}\left(\mathbf{r}_{1}\right)= & \frac{1}{2} \int d \mathbf{r}_{2} n\left(\mathbf{r}_{2}\right) H\left(r_{12}-d\right)\left(\Phi_{\alpha \beta}^{p}\left(\mathbf{r}_{12}\right)\right. \\
& \left.+\left.\frac{3}{5} a \frac{\partial \Phi_{\alpha \beta}^{p}}{\partial x_{\gamma}}\right|_{\mathbf{r}=\mathbf{r}_{12}}\left[p_{\gamma}\left(\mathbf{r}_{2}\right)-p_{\gamma}\left(\mathbf{r}_{1}\right)\right]\right) \\
& \times p_{\alpha}\left(\mathbf{r}_{1}\right) p_{\beta}\left(\mathbf{r}_{2}\right)
\end{aligned}
$$

can be, respectively, regarded as the contributions to the free energy per molecule due to the intermolecular interactions $\phi^{\text {att }}\left(r_{12}\right)$ and $\phi^{p}(1,2)$. Figure 12 shows these quantities as functions of $r_{1} . \omega^{p}$ can be shown to scale as $\chi p_{0} / q d$. When

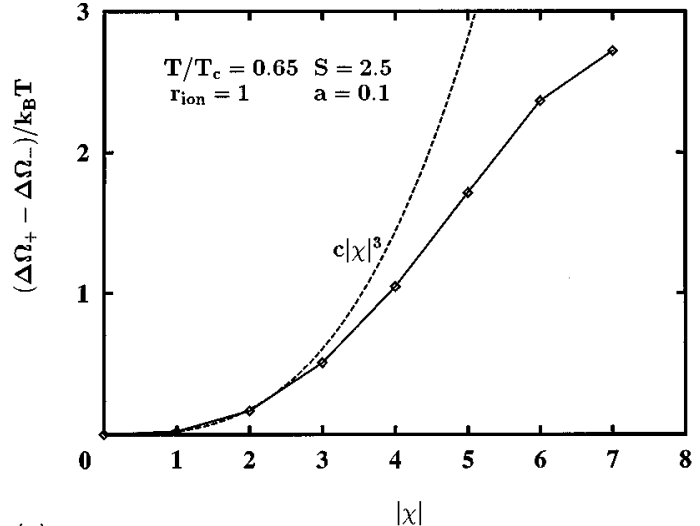

(a)

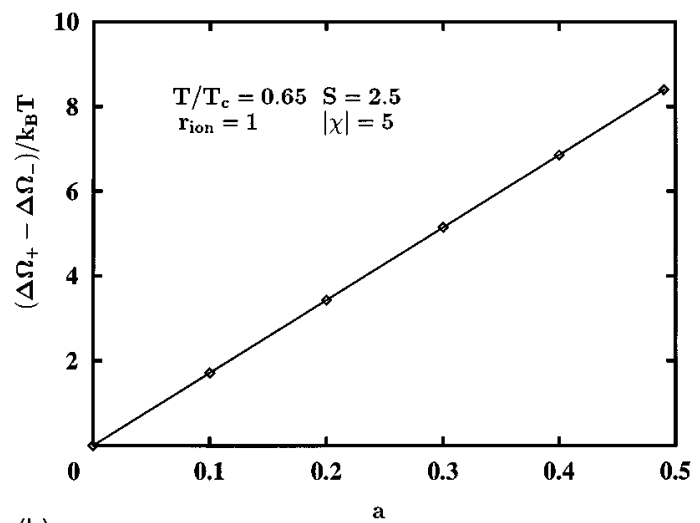

(b)

FIG. 11. (a) Dependence of the sign effect of the reversible work on $|\chi|$. The value of the coefficient $c$ is determined to match the computed value of $\left(\Delta \Omega_{+}-\Delta \Omega_{-}\right) / k_{B} T$ at $|\chi|=1$. (b) Dependence of the sign effect of the reversible work on $a$. Distance is normalized by $d$.

$p_{0}=1 \mathrm{D}, d=3 \AA$, and $q=e$, we have $p_{0} / q d \approx 0.07$. In Fig. 12 , we have chosen $p_{0} / q d=0.1$. The contribution due to $\phi^{p}(1,2)$ is, in fact, quite small compared to that from $\phi^{\text {att }}\left(r_{12}\right)$, justifying our approximations of ignoring the dipole-dipole interactions.

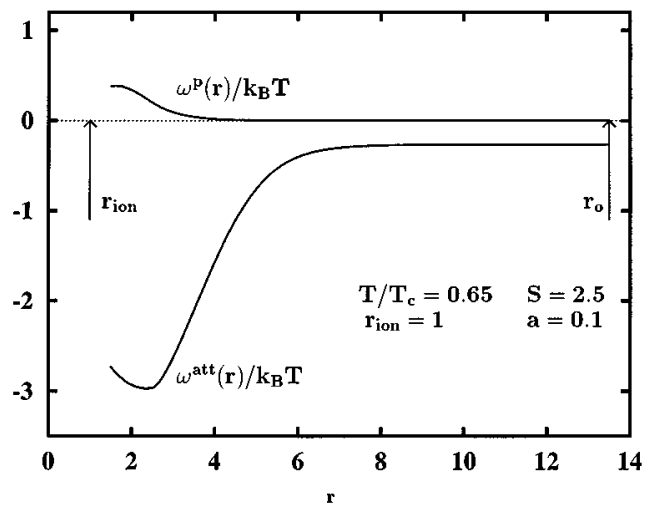

FIG. 12. The free energy densities resulting from the dipole-dipole interaction $\left(\omega^{p}\right)$ and that due to $\phi^{\text {att }}$ (denoted by $\left.\omega^{\text {att }}\right) . r_{0}$ is the radius of the system boundary. Distance is normalized by $d$. 


\section{DISCUSSION AND CONCLUSION}

In this paper, we have shown that the sign preference in ion-induced nucleation can be explained in terms of the asymmetric nature of the molecular interactions. Consistent treatment of such molecular characteristics is achieved by means of a statistical mechanical density functional theory, by which the grand potential is given in terms of two order parameters, the number density $n(r)$ of molecules, and the average dipole moment distribution $p(r)$. When the intensive state of the supersaturated vapor is specified, the stationarity condition of the grand potential uniquely determines a critical nucleus and a metastable nucleus for given values of model parameters. The calculated reversible work shows a preference toward the case of $\chi<0$, influencing the nucleation rate by a factor of $10-10^{2}$ for some realistic values of the model parameters employed in this paper.

In the present work, ions are assumed to be the only source of the electric field within the system. A uniform electric field is often applied, however, to selectively introduce ions with a particular sign. Rabeony and Mirabel $^{5}$ were the first to draw attention to the effect of a uniform electric field on the rate of nucleation. They reported a decrease in the number of nucleation events as the applied field was increased. A similar decrease was reported by Katz et al., ${ }^{21}$ though they clearly demonstrated that the nucleation rate, which was identified as the number of nucleation events divided by the time during which ions stay in the nucleation zone, was independent of the applied field. On the other hand, an increase in the nucleation events was reported by $\mathrm{He}$ and Hopke ${ }^{22}$ and El-Shall and Kane. ${ }^{23}$ Although a thermal diffusion cloud chamber was used in all of these experiments, the disagreement among them seems to arise from the different techniques of introducing ions into the nucleation zone.

In the experiments reported by Rabeony and Mirabel, ${ }^{5}$ a sign effect is observed only in the presence of this uniform field. However, Adachi et al. ${ }^{6}$ observed an apparent sign effect in the absence of the field. Their Fig. 9 indicates that the sign preference in the nucleation rate becomes less significant as supersaturation increases, in agreement with our prediction [see Fig. 7(b)]. Such dependence of the sign preference on supersaturation is also reported by El-Shall and Kane. $^{23}$

In trying to make quantitative comparisons with experiment, we note the following points. First, model parameters such as $\epsilon$ and $d$ are easily determined from the critical point data, assuming that the substance is well described by the law of corresponding states with two parameters. The dipole moment $p_{0}$ can be either related to the bulk dielectric constants or found in a handbook. The value of $a$ that plays such an important role in producing the sign effect, however, is not available in general.

Second, ions present in the experiments are often complex molecules such as $\mathrm{H}^{+}\left(\mathrm{H}_{2} \mathrm{O}\right)_{n}$ rather than ionized atoms. Then, the ion itself must be treated by means of statistical mechanics. Also, an ion would interact with the condensing molecules via a van der Waals type interaction as well. The latter can be treated in the current theory by simply replacing Eq. (19) by the appropriate expression.
We have characterized an ion by its charge and radius, and the ion-molecule interaction is assumed to be purely electrostatic. Such an idealization is acceptable for those ions that have the same electronic structure as that of noble gases. $^{24,25}$ On the other hand, one would not expect the present theory to be applicable to a system where the chemical nature of the interaction between the ion and molecules plays an important role. ${ }^{26}$ If, however, the effect on the grand potential due to such chemical characteristics is insensitive to the size of a nucleus, it will cancel out on calculating the reversible work of nucleation. In fact, Katz et al. ${ }^{21}$ found that, within the accuracy of their experiment, the nucleation rate dependence on the ion characteristics is insignificant. It is therefore interesting to examine the validity of the current theory against the experimental data, assuming that values of all the model parameters are known.

We have avoided the explicit consideration of the fluctuation of an ion within the nucleus by taking the position of the point charge as the origin. Implicit in our calculation is the assumption that the system is, on average, spherically symmetric around the ion. More rigorously, one could treat the system as a binary in which the ion is the second component with extremely low concentration.

Our calculations at this stage are largely qualitative both in the model representation of the system and in the theoretical treatment. Further improvement on both fronts can be envisaged. For example, the model can incorporate the polarizability of molecules, nonspherical charge distribution in an ion, etc. Also, a better treatment of the pair distribution function than that of Eq. (15) will undoubtedly give a better description of the structure of the fluid near the ion or interface. Nevertheless, it is clear that some of the most important characteristics of ion-induced nucleation have been captured in the present theory, which forms a basis for explaining this well known phenomenon that has hitherto remained inexplicable within the classical framework.

\section{ACKNOWLEDGMENT}

This work was supported by National Science Foundation Grant No. ATM-9307603.

${ }^{1}$ C. T. R. Wilson, Phil. Trans. R. Soc. London Ser. A 189, 265 (1897).

${ }^{2}$ L. B. Loeb, A. F. Kip, and A. W. Einarsson, J. Chem. Phys. 6, 264 (1938).

${ }^{3}$ L. Scharrer, Ann. Phys. 35, 619 (1939).

${ }^{4}$ D. R. White and J. L. Kassner, Jr., Aerosol. Sci. 2, 201 (1971).

${ }^{5}$ H. Rabeony and P. Mirabel, J. Phys. Chem. 91, 1815 (1987).

${ }^{6}$ M. Adachi, K. Okuyama, and J. H. Seinfeld, J. Aerosol Sci. 23, 327 (1992)

${ }^{7}$ J. J. Thomson, Conduction of Electricity through Gases (Cambridge University Press, Cambridge, 1906).

${ }^{8}$ L. Y. Chan and V. A. Mohnen, J. Atm. Sci. 37, 2323 (1980).

${ }^{9}$ S. H. Suck, J. Chem. Phys. 75, 5090 (1981).

${ }^{10}$ A. I. Rusanov and F. M. Kuni, J. Colloid Interface Sci. 100, 264 (1984).

${ }^{11}$ A. K. Shchĕkin, A. I. Rusanov, and F. M. Kuni, Kolloidn. Zh. 46, 535 (1984).

${ }^{12}$ H. Rabeony and P. Mirabel, J. Chim. Phys. 83, 219 (1986).

${ }^{13}$ H. Reiss, A. Tabazadeh, and J. Talbot, J. Chem. Phys. 92, 1266 (1990).

${ }^{14}$ H. M. Ellerby, C. L. Weakliem, and H. Reiss, J. Chem. Phys. 95, 9209 (1991).

${ }^{15}$ H. M. Ellerby and H. Reiss, J. Chem. Phys. 97, 5766 (1992).

${ }^{16}$ D. W. Oxtoby and R. Evans, J. Chem. Phys. 89, 7521 (1988).

${ }^{17}$ X. C. Zeng and D. W. Oxtoby, J. Chem. Phys. 95, 5940 (1991).

${ }^{18}$ R. Evans, Adv. Phys. 28, 143 (1979). 
${ }^{19}$ N. F. Carnahan and K. E. Starling, J. Chem. Phys. 51, 635 (1969); 53, 600 (1970).

${ }^{20}$ A. W. Castleman, Jr., P. M. Holland, and R. G. Keesee, J. Chem. Phys. 68, 1760 (1978).

${ }^{21}$ J. L. Katz, J. A. Fisk, and V. M. Chakarov, J. Chem. Phys. 101, 2309 (1994).

${ }^{22}$ F. He and P. K. Hopke, J. Chem. Phys. 99, 9972 (1993).
${ }^{23}$ M. S. El-Shall and D. Kane, presented at the 68th ACS Colloid and Surface Science Symposium, Stanford University, 19-22 June 1994 (unpublished).

${ }^{24}$ K. G. Spears, J. Chem. Phys. 57, 1850 (1972).

${ }^{25}$ I. N. Tang, H. R. Muckelwitz, and A. W. Castleman, Jr., Nature 230, 175 (1971).

${ }^{26}$ A. W. Castleman, Jr., Adv. Colloid Interface Sci. 10, 73 (1979). 